\title{
Leitlinien der DGVS
}

\begin{tabular}{|c|c|c|}
\hline Leitlinie & Erscheinungsjahr & Status \\
\hline \multicolumn{3}{|l|}{ Oberer Gastrointestinaltrakt } \\
\hline Zöliakie & 2014 & wird aktualisiert \\
\hline Refluxkrankheit & 2005, 2014 & wird aktualisiert \\
\hline Helicobacter pylori & 2009,2016 & gültig bis $07 / 2020$ \\
\hline \multicolumn{3}{|l|}{ Unterer Gastrointestinaltrakt } \\
\hline Chronische Obstipation & 2013 & wird aktualisiert \\
\hline Divertikelkrankheit & 2014 & wird aktualisiert \\
\hline Morbus Crohn & $2008,2011,2014$ & wird aktualisiert \\
\hline Reizdarmsyndrom & 2011 & Publikation 2019 \\
\hline Intestinale Motilitätsstörungen & 2011 & Publikation 2019 \\
\hline Colitis ulcerosa & 2011, 2018, 2019 & gültig bis $05 / 2023$ \\
\hline \multicolumn{3}{|l|}{ Leber, Galle, Pankreas } \\
\hline Hepatitis B & 2007,2011 & Publikation 2019 \\
\hline Lebertransplantation & & Publikation 2021 \\
\hline Akute und chronische Pankreatitis & 2012 & Publikation 2020 \\
\hline $\begin{array}{l}\text { Nicht-alkoholische Fettlebererkran- } \\
\text { kungen }\end{array}$ & 2015 & gültig bis $02 / 2020$ \\
\hline Hepatitis C & $\begin{array}{l}\text { 2009, Addenda 3/2014, } \\
6 / 2014,9 / 2014,2 / 2015 \text {, } \\
12 / 2016\end{array}$ & gültig bis $12 / 2022$ \\
\hline Behandlung von Gallensteinen & 2007,2018 & gültig bis $11 / 2022$ \\
\hline Autoimmune Lebererkrankungen & 2017 & gültig bis $02 / 2022$ \\
\hline Komplikationen der Leberzirrhose & 2011, 2019 & gültig bis $05 / 2023$ \\
\hline \multicolumn{3}{|c|}{ Maligne Erkrankungen des Gastrointestinaltrakts } \\
\hline Hepatozelluläres Karzinom & 1999, 2013 & wird aktualisiert \\
\hline Exokrines Pankreaskarzinom & 1999, 2001, 2006, 2013 & wird aktualisiert \\
\hline Magenkarzinom & $2002,2004,2012$ & Publikation 2019 \\
\hline Neuroendokrine Tumoren & 2018 & gültig bis $03 / 2023$ \\
\hline Ösophaguskarzinom & 2015,2018 & Gültig bis $12 / 2023$ \\
\hline Kolorektales Karzinom & 2004, 2008, 2012, 2017, 2019 & gültig bis $01 / 2024$ \\
\hline
\end{tabular}

Ә Open Access. (C) 2019 Frank Lammert, Petra Lynen Jansen, Markus M. Lerch, publiziert von De Gruyter.

(c) BY-NC-ND Dieses Werk ist lizenziert unter der Creative Commons Attribution-NonCommercial-NoDerivatives 4.0 Lizenz. https://doi.org/10.1515/9783110642513-205 


\begin{tabular}{|c|c|c|}
\hline Leitlinie & Erscheinungsjahr & Status \\
\hline \multicolumn{3}{|l|}{ Infektionen } \\
\hline $\begin{array}{l}\text { Gastrointestinale Infektionen und } \\
\text { Morbus Whipple }\end{array}$ & 2015 & gültig bis $01 / 2020$ \\
\hline \multicolumn{3}{|l|}{ Notfälle } \\
\hline Gastrointestinale Blutungen & 2017 & gültig bis $05 / 2022$ \\
\hline \multicolumn{3}{|l|}{ Gastrointestinale Endoskopie } \\
\hline $\begin{array}{l}\text { Qualitätsanforderungen in der gastro- } \\
\text { intestinalen Endoskopie }\end{array}$ & 2015 & gültig bis $07 / 2020$ \\
\hline $\begin{array}{l}\text { Sedierung in der gastrointestinalen } \\
\text { Endoskopie }\end{array}$ & 2008,2015 & gültig bis $05 / 2020$ \\
\hline
\end{tabular}

Alle Leitlinien der DGVS stehen unter www.dgvs.de kostenfrei zum Download zur Verfügung. 